\title{
Online medical consultation: a review
}

\author{
Ajeet Pal Singh ${ }^{1}$, Hari Shanker Joshi ${ }^{1 *}$, Arun Singh ${ }^{1}$, Medhavi Agarwal ${ }^{1}$, Palveen Kaur ${ }^{2}$
}

\author{
${ }^{1}$ Department of Community Medicine, Rohilkhand Medical College and Hospital, Bareilly, Uttar Pradesh, India \\ ${ }^{2}$ Indian Institute of Public Health, Delhi, PHFI, Gurgaon, Haryana, India
}

Received: 29 January 2018

Revised: 07 March 2018

Accepted: 08 March 2018

\author{
*Correspondence: \\ Dr. Hari Shanker Joshi, \\ E-mail: drjoshiharish@gmail.com
}

Copyright: ( $)$ the author(s), publisher and licensee Medip Academy. This is an open-access article distributed under the terms of the Creative Commons Attribution Non-Commercial License, which permits unrestricted non-commercial use, distribution, and reproduction in any medium, provided the original work is properly cited.

\begin{abstract}
The Internet has been a steady source of medical data previously; it has just as of late been utilized for online private patient-doctor consultations. As of late, the market has seen a surge in applications providing healthcare services on the go. An online consultation has secured a foothold in the market and individuals are opening up to the likelihood of substituting a visit to a physical facility with an online option. This study reports a review of the literature on online medical consultation from various databases as well as various surveys and reports published. As per published work/reports/surveys, the various factors which led to a sudden surge in the online medical consultation are the convenience, shift in disease patterns, cost-effective, privacy and second opinion.
\end{abstract}

Keywords: Virtual health, Telehealth, e-health, Telemedicine

\section{INTRODUCTION}

Online medical consultation (OMC) is the term utilized as a part of this paper to allude to web-based remote patient-specialist (consumer-provider) medicinal discussions. ${ }^{1}$ With the approach of broadband and video conferencing, numerous people have swung to online web-portals to get an online consultation. Utilization of this technological innovation has numerous advantages for both the doctor as well as the patient; including cost savings, comfort, accessibility, and enhanced privacy and communication. ${ }^{2}$ This idea is for patients with a variety of medical needs originating from different areas of a country or several nations. Patients may pick or be assigned to any specialist/general duty doctor who is accessible on the web. They are not limited to a particular care provider either by past learning or by geographical closeness. ${ }^{1}$
The aim of this review article is to explore the possible reasons behind the beginning of a new era of consultation, which is online medical consultation. It examines features and themes evident in the literature and in a range of currently operating platforms providing online medical consultation.

\section{METHODS}

\section{Study design}

The current study is a narrative review of published studies and articles.

\section{Databases}

Pub Med and Google Scholar.

Structured search strategy using appropriate keywords and MesH terms 


\section{FINDINGS FROM LITERATURE REVIEW}

\section{Convenience}

In the study named, reasons for consulting a doctor on the internet: web survey of users of an ask the doctor service, it was discovered that since computers are quite common, in homes as well as in working environment, in most developed nations it is quite evident why convenience was a noteworthy factor behind participants picking up Internet consultations. ${ }^{3}$ Moreover, the asynchronous access to the Internet-based Ask the doctor service enables users to get the services at any point of the day, a component which was applauded by many of the participants.

In a pilot study in South West England, it was noticed that patients were substantially more likely to utilize the system towards the beginning of the week. ${ }^{4}$ Out of all econsultations, $58.8 \%$ occurred from Monday to Wednesday, with usage declining towards the end of the week: just a $12.4 \%$ of e-consultations were done at the weekend. In any case, $69.9 \%$ happened in between 7 am and $5 \mathrm{pm}$, with peaks seen around 10 am and $2 \mathrm{pm}$. There was some enthusiasm for evening usage as well, but this was much lower than daytime utilization (19.4\% between $7 \mathrm{pm}$ and midnight, with a peak around $8 \mathrm{pm})$.

\section{A shift in disease patterns}

As per Sharma and Majumdar social story, over the period of 26 years, there has been a very obvious shift in the pattern of diseases across the country. ${ }^{5}$ In the $1990 \mathrm{~s}$, over $50 \%$ of the deaths were from communicable, maternal, neonatal, and nutritional diseases such as anemia, tuberculosis, and diarrhea. Though they have gradually reduced over the period of time, they still account for about $33 \%$ of all deaths in the country.

There has been an increasing incidence of noncommunicable diseases all over the globe. More than $50 \%$ of our population is now on the brink of dying from heart diseases, obesity, cancer, and diabetes. An increase of 25 percent in as many years. These diseases result in significant lifestyle modifications and require very different and unique forms of treatment, care, and adherence. With an ageing population, increasing urbanization and sedentary lifestyle this situation is further going to get worse putting an enormous burden on an already haphazard system.

The cost of treating communicable diseases in many of the cases is not high and results in one-time expenditure centered on specific incidence. However, the cost and complexity of managing chronic diseases are quite high resulting in abysmal screening and exceptionally poor adherence. For instance, the protocol recommended for screening of type 1 and 2 diabetes is 2-3 times a day and 3-4 times a week respectively as against our national average of 70 days plus per test.
As per the virtual visits consumer choice survey, $70 \%$ of patient respondents were of the view that they were willing to utilize virtual care for various types of visit. ${ }^{6}$ Online drug prescription, pre-surgical consultation, some post-operative appointments, receipt of oncology results, and chronic disease management check-ins were as the most preferred virtual care types.

As consumers increasingly look forward to convenient, affordable health care - and as payers' enthusiasm in lowcost access continues to grow aggressively - this survey suggests that consumers are most likely to shop for those who offer virtual visits for specialty and chronic care."

\section{Cost-effective}

In March 2009, President Obama identified "the biggest threat to our nation's balance sheet." Not major banks on the brink of insolvency. Not paralyzed credit markets. Not a bailout tab in the trillions. The biggest threat, he warned, "by a wide margin," is "the skyrocketing price of health care."

While the hospital charges you for its hygiene and maintenance, these royalty charges are not present in an e-visit since there is no infrastructure involved, cutting the cost of a consultation to much less or even half.

While an average doctor visit costs between 1000 to 1500 rupees, an online consult can turn out to be very economical with costs ranging from 50 to 500 rupees.

\section{Privacy}

The confidentiality level in an encrypted conversation with an online doctor is unmatched. The hospital will never have the capacity to give in-private consultation since you have to be available publicly in the crowd to see the doctor. Subsequently, though medical records are kept undisclosed in password protected systems and computers, the staff handling it is well versed with your details. However, in an e-consultation model, this is kept amongst you and your doctor alone with no other human interaction in between. So all data privacy and protection is maintained and remains very confidential.

In the internet consultation, the individual may stay unknown thereby enabling inquirers to ask, e.g., sensitive and embarrassing questions. More than $33 \%$ of the participants valued the chance of being able to ask anonymously, suggesting that this feature may supplement regular health care. ${ }^{1}$ In previous studies "health seekers" also appreciated the anonymity of searching the Internet for medical information. ${ }^{8,9}$

\section{Second opinion}

It was noticed that numerous participants expressed a need for a second opinion, which might be one of the significant highlights that Internet Ask the doctor services 
can provide. In Sweden, the privilege of a second opinion is allowed just on account of serious health conditions. For less-serious medical issues, or if there is a failure to communicate with the regular doctor, it is quite hard to get another doctor's evaluation of one's medical issues.

\section{Women being at home}

In a study conducted by Edwards et al (2017) it was seen that women were almost twice as likely to perform an econsultation as men $(64.7 \%$ vs. $35.3 \%)$ and over half of all users were age 25-44 years (median age 39). After the age of 45 years, use declined with age, although young adults (age 18-24 years) also accounted for only a small proportion of total usage $(8.7 \%){ }^{4}$

\section{Areas of concern}

- A failed telehealth experience may be a reason for absolute dissatisfaction for patients. It can fail in terms of internet connectivity on either side or in terms of a low level of care provided from doctor's side.

- Some specialties essentially are not helpful to virtual care, such as physical therapy or orthopedic followups.

- This model of care also raises the question of whether more medical test and investigations will be ordered in the absence of being able to physically conduct a physical examination of the patient.

- It also puts a doctor in the difficult situation of making a diagnosis without a proper understanding of the patient's medical history and social context, in addition to removing the opportunity to conduct a physical examination.

\section{Suggestions}

- A personal touch and rapport building during an online conversation between doctor-patient is the need of the hour.

- Instead of stating a diagnosis online, a list of differential diagnosis can be suggested to the patient. Prevention should be the main theme of discussion online instead of treatment.

- In case of non-availability of doctors or busy schedule, appropriate waiting time to be mentioned to the patients.

- A user-friendly interface so that the patient can attach all reports of the tests as well as previous treatment records including prescriptions since it will help the doctor to give you more appropriate advice.
Funding: No funding sources Conflict of interest: None declared

Ethical approval: Not required

\section{REFERENCES}

1. Ibrahim Al-Mahdi, Kathleen Gray, Reeva Lederman. Online Medical Consultation: A review of literature and practice, 2015. Available at: http://crpit.com/confpapers/CRPITV164Al-

Mahdi.pdf. Accessed on 6 March, 2018

2. Janet B. Prince. Health care crisis in America. 6th ed. New York, NY: Nova Science Publishers, Inc; 2006.

3. Umefjord G, Petersson G, Hamberg K. Reasons for Consulting a Doctor on the Internet: Web Survey of Users of an Ask the Doctor Service. J Med Internet Res. 2003;5(4):e26.

4. Edwards HB, Marques E, Hollingworth W, Horwood J, Farr M, Bernard E, et al. Use of a primary care online consultation system, by whom, when and why: evaluation of a pilot observational study in 36 general practices in South West England BMJ Open. 2017;7:e016901.

5. Sharma M, Majumdar D. Disease patterns in India are shifting; are entrepreneurs prepared to fix healthcare in India? 2017. Available at: https://yourstory.com/2017/11/disease-patternsindia/. Accessed on 20 January 2018.

6. Zuehlke E. What do Consumers Want from Virtual Visits? 2017. Available at: https://www.advisory. com/research/market-innovation-center/researchbriefs/2017/virtual-visits-briefing. Accessed on 20 January 2018.

7. Slater C. The Doctor of the Future, 2013. Available at: http://www.fastcompany.com/1266043/doctorfuture. Accessed 20 January 2018.

8. Susannah F. The online health care revolution: How the web helps Americans take better care of themselves. Washington, DC: The Pew Internet \& American LifeProject; 2000. Available at: http://www.pewinternet.org/reports/toc.asp?Report= 26. Accessed 20 January 2018.

9. Rideout V. Generation Rx.com: how young people use the Internet for health information. 2001. Available at: http://www.kff.org/content/2001/ 20011211a/GenerationRx.pdf. Accessed 20 January, 2018.

Cite this article as: Singh AP, Joshi HS, Singh A, Agarwal M, Kaur P. Online medical consultation: a review. Int J Community Med Public Health 2018;5:1230-2. 